\title{
特集＼cjkstart手術のための血管解剖を極める
}

\section{Functional Venous Anatomy of the Brain for Neurosurgeons}

\author{
Masaki Komiyama, M.D. \\ Department of Neuro-Intervention, Osaka City General Hospital
}

A thorough knowledge of the functional vascular anatomy of the brain is becoming increasingly required in neuro-interventional procedures. Similarly, this knowledge is also required in neurosurgery, especially when a particular artery or vein is to be sacrificed during surgery. Permanent occlusion of a major artery can be challenged by a balloon occlusion test, but for venous sacrifice, such a procedure is practically not applicable. Up to now, reliable methods to judge the safety of such destructive procedures were lacking. Knowledge of the basic angioarchitecture of the cerebral veins, in other words, the "functional anatomy of the cerebral veins" may help us to better understand the safety or risk of sacrificing cerebral veins. Today, 3D-CT angiography, digital subtraction angiography and cone-beam CT provide detailed information on the precise cerebral venous anatomy to help us understand the functional venous anatomy and to make informed decisions.

(Received December 19, 2016 ; accepted January 17, 2017)

Key words : functional venous anatomy, collateral circulation, ischemic complication, neurosurgery, occlusion of cerebral veins

Jpn J Neurosurg（Tokyo）26:488-495, 2017

\section{Introduction}

The intentional occlusion of a particular cerebral vein is sometimes required during neurosurgical procedures. However, the safety of such venous sacrifice is not adequately appreciated. Furthermore, a variety of factors including intraoperative manipulation, i.e. compression of the brain and retraction of veins, make evaluation of the tolerance of the regional venous occlusion more complicated. In endovascular procedures, intentional venous occlusion is usually performed to treat a dural arteriovenous malformation, which has already caused regional venous hypertension and/or leptomeningeal reflux, thus allows safe sacrifice.

For robust endovascular procedures, knowledge of the functional vascular anatomy of the brain is essential ${ }^{14) 15)}$. "Dangerous (arterial) anastomoses" are of well-known arterial anatomy, indicating apparent or dormant anastomoses between the external carotid system, and the internal carotid system and/or vertebra-basilar system. Representative dangerous anastomoses include the cavernous branch of the middle meningeal artery connecting to the inferolateral trunk of the internal carotid artery, and the mastoid branch of the occipital artery connecting to the vertebral artery. Similarly, in the neurosurgical field, there are "dangerous veins" which cannot always be sacrificed safely ${ }^{1)}$. Those include the vein of Labbè, the superficial Sylvian vein, the superior petrosal vein, the dominant transverse sinus, the posterior two-third of the superior sagittal sinus, the straight sinus and the torcular herophili ${ }^{19) 34) 35)}$.

For understanding and prevention of neurological adverse sequels after surgical venous sacrifice, the author reviewed the reported success and failure of such procedures in the neurosurgical literature, and discusses possible

Address reprint requests to: Masaki Komiyama, M.D., Department of Neuro-Intervention, Osaka City General Hospital, 2-1322 Miyakojima-Hondori, Miyakojima, Osaka 534-0021, Japan 
solutions to avoid adverse sequels of venous sacrifice.

\section{Surgical sacrifice of the cerebral veins in the literature}

\section{Superficial cortical veins including the super- ficial Sylvian vein and the vein of Labbè}

Sacrifice of superficial Sylvian veins is often tolerated, but this is not always the case $\mathrm{e}^{19)}$. It is well known that injury to the lateral tentorial sinus, above all, the vein of Labbè during temporal approach may cause serious complications with severe brain swelling and hemorrhage of the temporal lobe, indicating intraoperative sacrifice of the lateral tentorial sinus being a high-risk procedure.

\section{Thalamostriate vein (terminal vein)}

The thalamostriate vein, also known as a terminal vein, was occasionally sacrificed in the frontal approach for the third ventricle tumor to obtain a wide view through the foramen of $\mathrm{Monro}^{33)}$. The sequels of such venous sacrifice varied from uneventful to the severe consequence of basal ganglia infarction ${ }^{9) 21}$. Possible collaterals after occlusion of the thalamostriate vein might be the transcerebral venous route as well as the terminal venous arcade (superior terminal vein-middle terminal vein-inferior terminal vein-inferior ventricular vein). This terminal venous arcade runs parallel to the stria terminalis between the caudate nucleus and thalamus (Fig. 3).

\section{Great vein of Galen}

In the early twentieth century, experimental occlusion of the great vein of Galen in animals was performed by many neurosurgeons to create "experimental hydrocephalus" ${ }^{3) 6(32)}$. Two of 9 rhesus monkeys were lethal within two days after sacrifice of the vein ${ }^{3)}$. This occlusion of the great vein of Galen failed to create hydrocephalus, but in turn, it resulted in various sequels due to variable, individual venous collaterals, i.e., anastomoses between the basal vein and transverse sinus. Similarly, occlusion of the great vein of Galen in humans resulted in variable results due to the same reasons of variable venous anatomy ${ }^{34)}$.

\section{Superior petrosal vein}

The superior petrosal vein is known also as "petrosal vein of Dandy” named after Walter E. Dandy [1886-1946] thanks to his work especially in posterior fossa surgery ${ }^{5)}$. He called special attention to this vein during operations on trigeminal nerves. This vein drains the anterior aspect of the cerebellum and brain stem. Its tributaries include the vein of cerebellopontine fissure, the vein of middle cerebellar peduncle, the transverse pontine vein, the pontotrigeminal vein, and veins draining the lateral cerebellar hemisphere, among which the vein of cerebellopontine fissure is the larges $\mathrm{t}^{29)}$. The superior petrosal vein was sometimes sacrificed in more than 4,400 cases of microvascular decompression procedures by Peter Joseph Janetta [1932-2016] without any sequels ${ }^{2223)}$. Classically, occlusion of draining veins to the superior petrosal vein during acoustic neurinoma removal is safely performed, which was described in the famous textbook by Ludwig G. Kempe $\left[1915^{-2012}\right]^{11)}$. However, there are several reports of mild and severe ischemic complications including pontine and/or cerebellar hemorrhagic infarction, and peduncular hallucinosis (visual hallucinations associated with brainstem lesions) resulting from severing the superior petrosal vein ${ }^{132} 20$ )25)33) 34)39).

\section{Precentral cerebellar vein}

In the infratentorial supracerebellar approach, which was originally described by Fedor Krause [1856-1937] in 1926, it is necessary to divide some of the bridging veins, which include superior/inferior hemispheric veins, vermian veins, and the vein of cerebello-mesencephalic fissure (precentral cerebellar vein ${ }^{18)}$. Bennett M. Stein [1931-] revived this approach without any sequels in 6 cases in $1971^{36)}$. When the superior vermian vein drains into the precentral cerebellar vein, the united vein is called the superior cerebellar vein. It is recommended to divide the superior cerebellar vein away from the great vein of Galen to minimize the flow compromise ${ }^{12)}$. These veins are the major veins belonging to either the superior or posterior drainage routes of the cerebellum. In most cases, intentional occlusion of these veins was feasible ${ }^{28) 34) 40}$. However, cerebellar swelling after sacrifice of the lateral bridging vein (probably the superior petrosal vein group) in addition to medial bridging veins leading to death on day 2 was also reported ${ }^{27)}$.

\section{Transverse sinus}

The transverse sinus was sometimes severed between the entry point of the vein of Labbè and superior petrosal 
sinus to gain a wider operative field in operations involving posterior fossa pathology ${ }^{19}$. In the case of intentional sacrifice of the transverse-sigmoid sinus, dominancy of the venous flow in the transverse sinuses is important. Inappropriate sacrifice of the dominant transverse sinus may result in catastrophic sequels.

\section{Prediction and prevention of the adverse sequels of venous sacrifice}

\section{Factors influencing the results of venous sac- rifice}

The most important factor whether the venous sacrifice is possible or not is an existence or establishment of sufficient collaterals or rerouting when venous sacrifice is performed. In addition, the following are also important factors : positioning which defines the basic venous pressure, intraoperative removal of cerebrospinal fluid and/or ventricular drainage if necessary which reduces the chance to retract the brain, surgical manipulations including traction of veins which compromise the venous flow, compression of the brain by a spatula, intraoperative hypotension, and thrombosis formation around the sacrificed vein. Age is also related to tolerance of venous sacrifice because of high thrombogenecity in the elderly ${ }^{26)}$. Post-operative management may also influence the results of venous sacrifice. These are the factors that more or less contribute to the outcome of the surgical sacrifice of the cerebral veins.

\section{Monitoring the safety of venous sacrifice}

The simplest way to test tolerance of the venous occlusion is to temporary clamp the vein with microforceps or a temporary clip, and to observe the regional congestion for a few minutes ${ }^{34)}$. However, this method is subjective and not reliable. Intraoperative continuous monitoring of brainstem auditory evoked potentials was used during the test occlusion of the petrosal vein ${ }^{37)}$. The somatosensory evoked potential was also used for monitoring the occlusion of the Sylvian vein ${ }^{1)}$. Monitoring of these evoked potentials may predict possible venous ischemia, but false negative results might also occur. Measurement of the local cerebral blood flow might be a theoretical option ${ }^{2}$, which is difficult to perform and false negatives could occur. Ferroli et al. reported the intraoperative temporary occlusion of the cortical vein using indocyanine green (ICG), i.e.,"temporary
ICG clipping", to examine the venous collateral to determine whether a particular cortical vein can be sacrificed safely or not ${ }^{7)}$. After intraoperative temporary clipping of a target cortical vein, stagnation of ICG was interpreted as poor venous collateral, but non-stagnation was indicative of good collaterals, thus the vein might be safely sacrificed. In consideration of venous collaterals, their reliance on the presence or absence of the contrast stagnation could be misleading, and some cases without stagnation may occur, but the opposite situation may also hold true. Additionally, any of the above methods may not accurately predict the delayed complications of venous sacrifice.

\section{Prevention of the adverse effects of venous sacrifice}

One surgical technique, originally reported by Kenichirou Sugita [1932-1994], to avoid venous occlusion is dissection of a particular vein from the surrounding arachnoid and/or cortex, and removal of a small amount of the brain tissue around the vein in order to preserve the flow in the vein $^{38)}$. Also, brain compression, if necessary, must be intermittent with the least force and the shortest dura$\operatorname{tion}^{25}{ }^{34}$. Compression to the brain of more than $20 \operatorname{Torr}(=$ $\mathrm{mmHg}$ ) stops the venous return, which results in cerebral infarction if the compression is prolonged ${ }^{19)}$. When the bridging veins are to be severed, severing the vein as close to the dural entry as possible may preserve the venous flow in the remaining veins on the cortical side ${ }^{134)}$. Changing the exposure route (approach) and/or widening the bone window for skull base surgery are the other options to avoid the adverse venous sequels.

\section{Staged surgery for the expected develop- ment of venous collaterals}

Staged surgery is an idea which Leonard I. Malis [1919-2005] provided initially ${ }^{19)}$, whereby surgical ligation of the outflow of the Sylvian vein alone is accomplished in the first operation, which is then followed by definite transor subtemporal surgery for the removal of the skull base tumor. Such staged surgery has been reported to increase the tolerance of surgical manipulation after occlusion of the bridging vein ${ }^{31)}$. The second operation is performed about 2 weeks after the initial venous sacrifice. During this period, collaterals develop, anastomosing with the other draining routes. It is not always safe to sacrifice the Sylvian vein in 


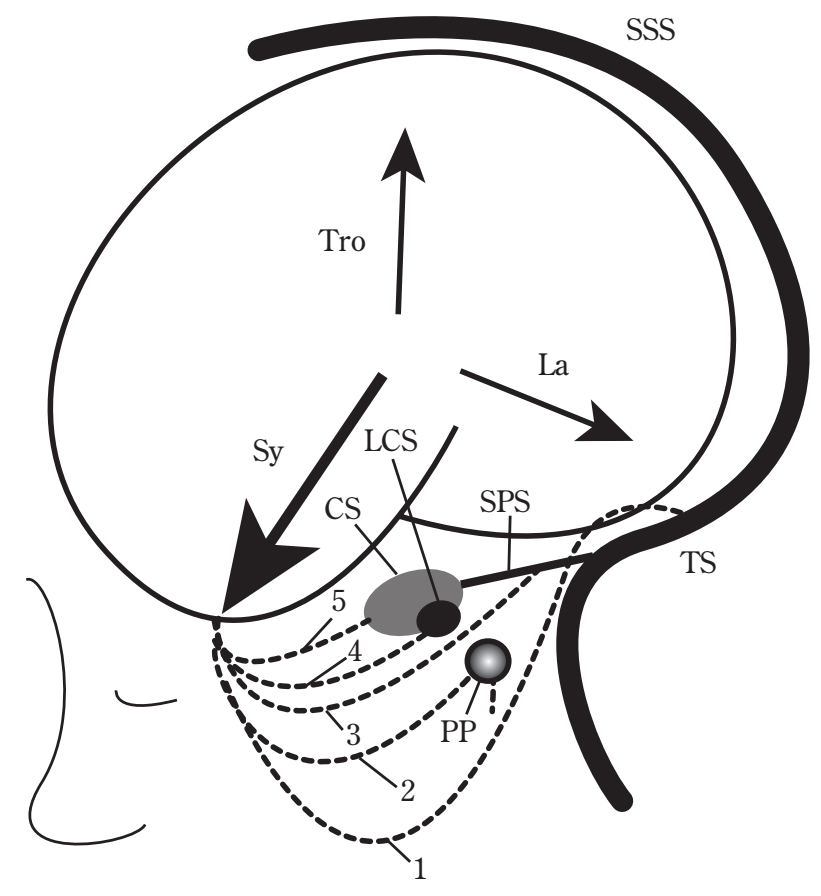

Fig. 1 Superficial cerebral veins and drainage patterns of the superficial middle cerebral vein

The vein of Trolard (Tro, frontoparietal vein), superficial Sylvian vein (Sy, superficial middle cerebral vein) and vein of Labbè ( $\mathrm{La}$, temporooccipital vein) have enough anastomoses between each other. Drainage patterns of the superficial middle cerebral vein are as follows : to the transverse sinus (TS) (Route 1), to the pterygoid plexus (PP) through the sphenobasal vein (Route 2), to the superior petrosal sinus (SPS) through the sphenopetrosal vein (Route 3 ), to the laterocavernous sinus (LCS) (Route 4), to the cavernous sinus (CS) (Route 5), or any combination thereof. Route 5 is the most mature form called the "cavernous sinus capture" of the superficial middle cerebral vein.

the initial step. It is true, that in extremely slow growing meningiomas, occluding the so-called dangerous veins may facilitate collaterals to develop or redirect the venous outflow. However, a duration of 2 weeks between two operations and the latent period of venous sinus occlusion by meningiomas are quite different clinical situations. Therefore, staged surgery is at present not a reliable method to avoid venous ischemia caused by sacrifice of the bridging vein.

\section{Reconstruction of the severed veins}

Reconstruction of the severed veins or sinuses is another possibility to prevent subsequent venous infarction,

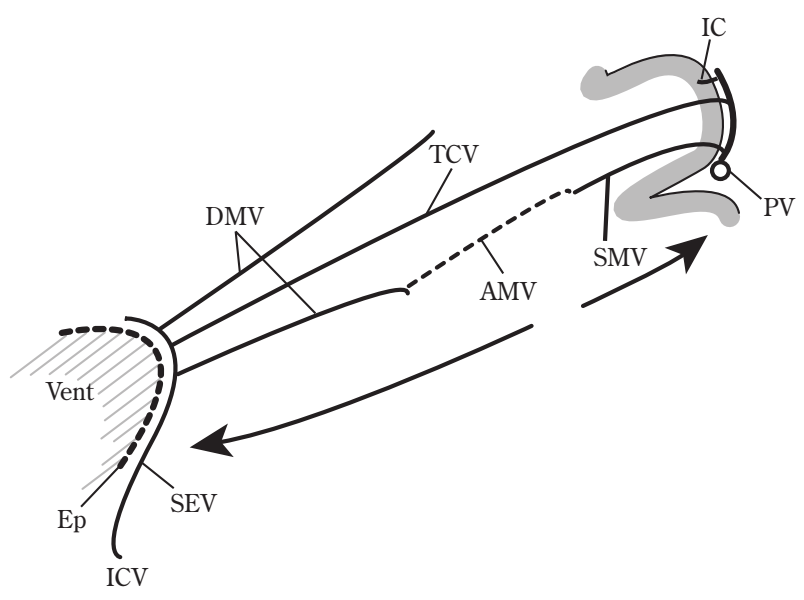

Fig. 2 Medullary veins

Medullary veins may contribute as collaterals between the supratentorial superficial and deep venous systems. There are four types of medullary veins : 1) the superficial medullary vein (SMV) and 2) the deep medullary vein (DMV), 3) the anastomotic medullary vein (AMV) between the SMV and DMV, and 4) the transcerebral vein (TCV) which connects the pial vein (PV) of the brain surface to the subependymal vein of the lateral ventricle. The TCV is the most primitive vein since this vein acts as the drainage from the germinal matrix in the early embryogenesis $^{17)}$. Arrows indicate basic flow patterns of the medullary veins.

Ep : ependyma, IC : intracerebral vein, ICV : internal cerebral vein, SEV : subependymal vein, Vent: ventricle

but not like a surgical anastomosis of the severed arteries, this procedure is extremely difficult for the veins. Accordingly, a limited number of such cases has been reported in the literature ${ }^{4) 10) 24) 30)}$.

\section{Insights from the functional venous anatomy}

From the preceding review of the neurosurgical literature, it can be summarized that the safety of the surgical venous sacrifice cannot be precisely predicted. Surgical manipulation including brain compression and traction of the vein, and postoperative management in terms of blood pressure and the coagulation system, further make prediction difficult. The only truly reliable way to prevent venous ischemia is to not sacrifice the vein.

However, venous sacrifice might be required in some occasions. Under these circumstances, what we can do is to estimate the possibility of the safety or risk of venous sacri- 


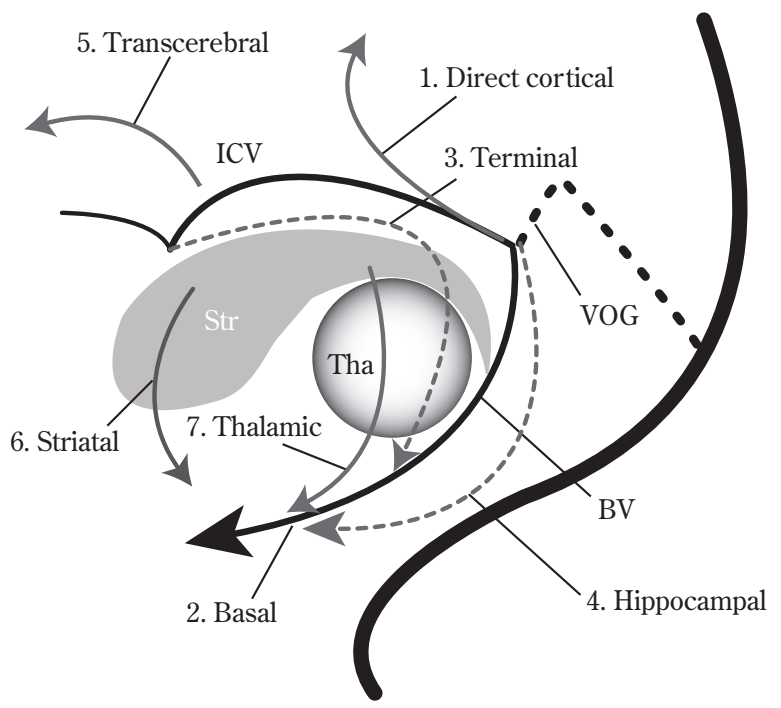

Fig. 3 Collateral pathways in case of occlusion of the vein of Galen and/or straight sinus

Collateral pathways from the deep venous system to the superficial venous system are as follows: 1 ) the direct cortical vein route (through the internal occipital vein and posterior pericallosal vein), 2) the basal vein route (to the cavernous sinus, etc), 3) the terminal arcade route, 4) the hippocampal vein route (through the posterior and anterior longitudinal hippocampal veins), and 5) the transcerebral vein routes. Among transcerebral routes, 6) the striatal (subpallial) and 7) thalamic (diencephalic) routes are prominent.

BV : basal vein, ICV : internal cerebral vein, Str : striatum, Tha : thalamus, VOG : vein of Galen

fice judging from the angiographic information, that is, the possibility of alternative drainage routes for the sacrificed vein ${ }^{19)}$. To predict the collaterals, a thorough understanding of the functional venous anatomy is helpful and mandatory. The following are some excerpts from the author's previous paper on functional venous anatomy of the brain ${ }^{15)}$.

\section{Imaging of the venous system}

With advances in neuro-imaging, especially MR imaging, information on various brain pathologies can be provided. As far as venous anatomy is concerned, $3 \mathrm{D}^{-\mathrm{CT}}$ venography, venous phase of digital subtraction angiography, the venous phase of 3D angiography, and cone-beam CT provide at present the most detailed views of venous anatomy. 3D angiographic images can also be viewed stereoscopically, a classic technique but one which still enhances our understanding of the detailed venous anatomy just as it did for our neuroradiological pioneers in 1960-

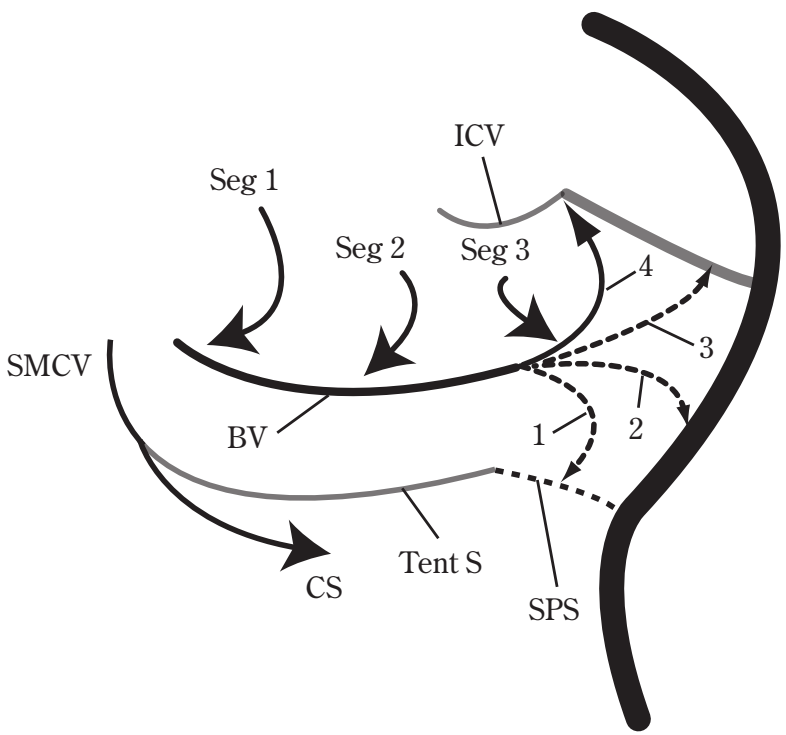

Fig. 4 Embryology of the basal vein of Rosenthal and its drainage patterns

Due to outgrowth of the prosencephalon, the stretched distal portion of the primitive tentorial sinus (Tent S) is obliterated. The venous return from the telencephalon, diencephalon and mesencephalon to the tentorial sinus are transferred to the newly formed longitudinal anastomoses of the basal vein (BV). The basal vein is composed of three segments, corresponding to the transverse veins of the telencephalon (Seg 1), diencephalon (Seg 2), and mesencephalon (Seg 3). Distal portions of the basal vein show the following variable drainage patterns: Route $1:$ to the superior petrosal sinus (SPS), Route $2:$ to the transverse sinus, Route $3:$ to the straight sinus or torcular herophili, and Route $4:$ to the vein of Galen or internal cerebral vein (ICV). Embryologically, Route 1 is the most primitive form.

CS : cavernous sinus, SMCV : superficial middle cerebral vein

$70 \mathrm{~s}^{16)}$.

\section{Supratentorial superficial venous system}

Superficial and deep Sylvian veins are composed of embryological superficial and deep telencephalic veins that drain to the primitive tentorial sinus initially. The superficial Sylvian vein is hemodynamically balanced with the anastomotic vein of Trolard superiorly and the anastomotic vein of Labbè inferiorly. Thus, these three drainage routes are well anastomosed and balanced. A particular cortical vein could be sacrificed under well-developed superficial anastomoses, but in case of poor collaterals, preservation of the cortical vein is necessary. Embryologically, after the occlusion of the primitive tentorial sinus posteriorly, the superficial Sylvian 


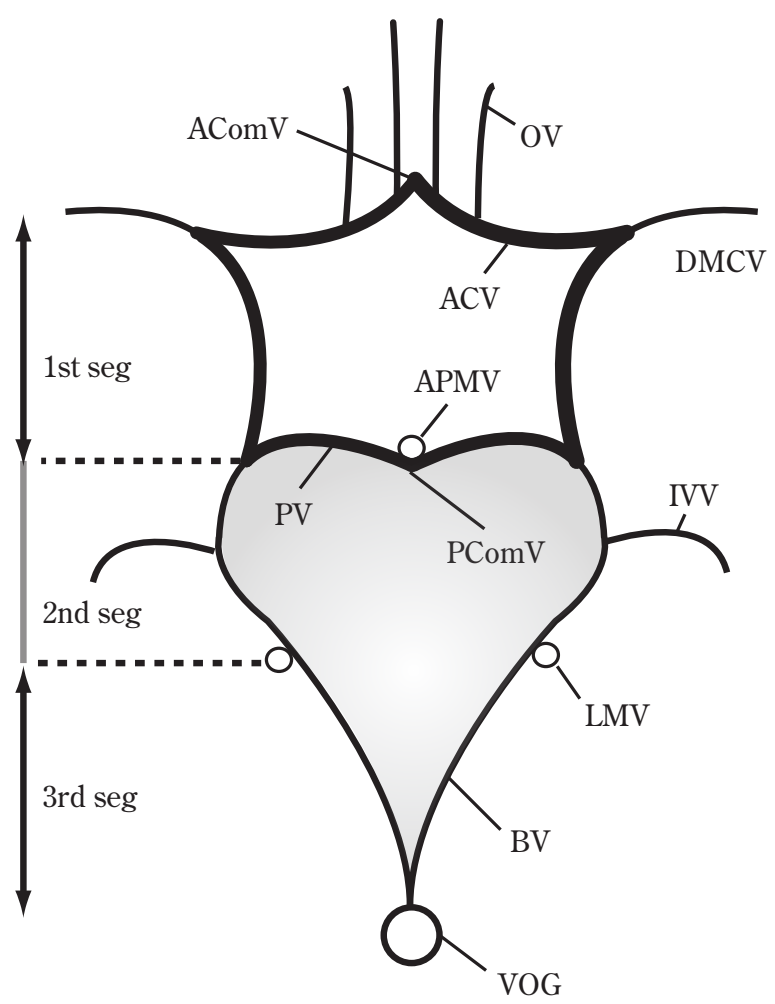

Fig. 5 Venous circle of Trolard and the three basal vein segments

Similar to the arterial circle of Willis, there is a venous circle of Trolard at the base of the brain. This circle provides transverse and longitudinal anastomoses at the skull base. The venous circle is shown by thick lines. The three segments of the basal vein are shown on the left side. Peduncular veins, the second and third segments of basal veins form the so-called "mesencephalic heart" (gray color).

AComV : anterior communicating vein, ACV : anterior cerebral vein, APMV : anterior pontomesencephalic vein, BV: basal vein, DMCV : deep middle cerebral vein, IVV : inferior ventricular vein, LMV : lateral anastomotic mesencephalic vein, OV: olfactory vein, $\mathrm{PComV}$ : posterior communicating vein, $\mathrm{PV}$ : peduncular vein, VOG : vein of Galen

vein has several choices as its drainage routes, in the order of embryological, thus phylogenetic primitiveness, to 1 ) the transverse sinus, 2) the pterygoid plexus through the foramen ovale (sphenobasal vein), 3) the superior petrosal sinus (sphenopetrosal vein), 4) the laterocavernous sinus, and 5) the cavernous sinus ${ }^{8)}$ (Fig. 1).

\section{Supratentorial deep venous system}

The deep venous system initially develops as a drainage route of the choroid plexus system, which provides nutrition to the early neural tube. Its drainage is through

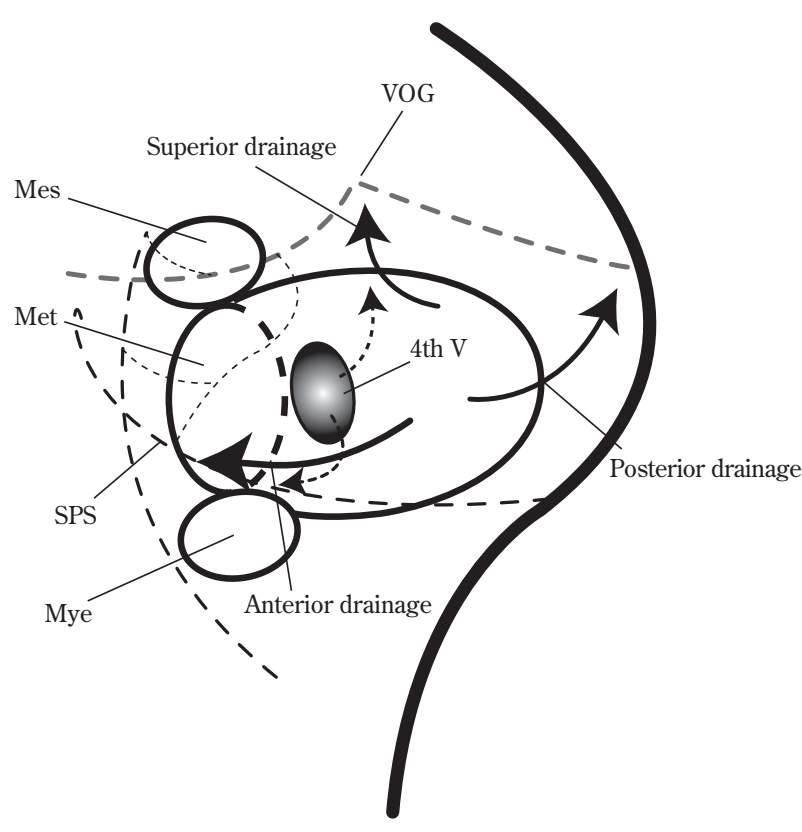

Fig. 6 Venous drainage routes of the brainstem and cerebellum

There are three basic drainage routes for the brainstem and cerebellum: 1 . Anterior drainage (to the superior petrosal vein), 2. superior drainage (to the vein of Galen), and 3. posterior drainage (to the transverse sinus and torcular herophili). Interrupted arrows indicate deep venous drainage from the subependymal veins to the precentral cerebellar vein superiorly and to the vein of the lateral recess of the fourth ventricle anteriorly.

Mes: mesencephalon, Met: metencephalon, Mye : myelencephalon, SPS : superior petrosal sinus, VOG : vein of Galen, 4th V: 4th ventricle

the median vein of prosencephalon at $6-11$ weeks of gestation. After cranial regression of this vein, the adult deep venous system is established. Parenchymal venous drainages are either to the superficial veins through the superficial medullary veins or to the deep venous system (subependymal vein) through the deep medullary veins. The anastomotic medullary vein connects them. Transcerebral veins connect to the superficial cerebral and deep subependymal veins directly. There are many variations in the anatomy of the subependymal veins ${ }^{33)}$, which enables various collaterals in the normal and abnormal settings (Fig. 2).

When the vein of Galen is occluded due to any causes or an $\mathrm{AV}$ shunt flows in this vein in the retrograde fashion, possible rerouted deep venous drainages are as follows: 1 ) The direct cortical vein route through the internal occipital and posterior pericallosal veins, 2) the basal vein route, 3 ) 
the terminal vein route through the terminal arcade, 4) the longitudinal hippocampal vein route, and 5) the transcerebral vein route. The terminal vein route and longitudinal hippocampal vein route are anastomoses between the internal cerebral vein and basal vein. Prominent transcerebral vein routes between the embryonic, dorsal, and ventral veins are 6) the striatal route in the striatum and 7) the thalamic route in the thalamus. The thalamic route is commonly observed after occlusion of AV shunts for the vein of Galen aneurysmal malformations (Fig. 3).

\section{Basal venous system}

Embryologically, after occlusion of the primitive tentorial sinus posteriorly, the basal vein of Rosenthal develops. This vein is a new vein (late acquisition) composed of three segments : the first segment (telencephalic), second segment (diencephalic), and third segment (mesencephalic). Disconnection between these segments is common, making various anatomical variations possible. The basal vein receives not only the deep venous drainage, but also the superficial venous drainage. Therefore, this vein can be regarded as the superficial vein of the skull base, which receives deep venous drainage as well. The basal vein may select one of the following draining routes. Embryologically and phylogenetically, in the order of primitiveness: the basal vein drains to the superior petrosal sinus (Route 1), the transverse sinus (through tentorial sinus) (Route 2), the straight sinus (Route 3), and the great vein of Galen (Route 4) (Fig. 4, 5).

\section{Infratentorial venous system}

Essentially, there are three routes draining the brainstem and cerebellum ${ }^{29)}$. Anterior drainage (superior petrosal drainage) drains the archicerebellum, which is the basic drainage of the cerebellum through the embryological ventral metencephalic vein (trigeminal vein). Superior drainage (Galenic drainage) drains the paleocerebellum, and posterior drainage (torcular drainage) drains the neocerebellum. There are abundant transverse (transverse pontine veins) and longitudinal (anterior pontomesencephalic vein, anterior medullary veins, anastomotic lateral mesencephalic vein) anastomoses superficially between the three drainage routes. Like the cerebrum, the cerebellum has superficial and deep venous systems. Subependymal veins around the fourth ventricle drain to the precentral cerebellar vein and the vein of the lateral recess of the fourth ventricle, both of which also receive the superficial cerebellar veins. Subependymal veins under the floor of the fourth ventricle drain anteriorly through the anterior and lateral transpontine veins (Fig. 6).

\section{Conclusion}

Up to now, there have been no reliable methods to predict the safety of the sacrificing procedure of a particular vein. For neurosurgeons, every effort to preserve the veins is the best way to avoid unsure risk of venous sacrifice. When a particular vein is to be sacrificed, the functional venous anatomy with an emphasis on collaterals should be contemplated on the individual basis to predict safety of such venous sacrifice.

COI : The author has nothing to declare.

\section{References}

1) $\mathrm{Al}-$ Mefty $\mathrm{O}$, Krisht $\mathrm{AF}$ : The dangerous veins. in Hakuba $\mathrm{A}$ (ed) : Surgery of the Intracranial Venous System: Embryology, Anatomy, Pathophy Siology, Neuroradiology, Diagnos Treatment. New York, Springer, 1996, pp.338-345.

2) Arbit E, DiResta GR, Bedford RF, Shah NK, Galicich JH : Intraoperative measurement of cerebral and tumor blood flow with laser-doppler flowmetry. Neurosurgery $\quad \mathbf{2 4}: 166^{-}$ 170, 1989 .

3) Bedford THB : The venous system of the velum interpositum of the rhesus monkey and the effect of experimental occlusion of the great vein of Galen. Brain $\quad \mathbf{5 7}: 255-265$, 1934.

4) Bonnal J, Brotchi J : Surgery of the superior sagittal sinus in parasagittal meningiomas. J Neurosurg $\quad 48$ : 935-945, 1978

5) Dandy WE : An operation for the cure of tic douloureux : partial section of the sensory root at the pons. Arch Surg 18: 687-734, 1929 .

6) Dandy WE, Blackfan KD : An experimental, clinical and pathological study. Part 1. Experimental studies. Am J Dis Child 8: 406-482, 1914

7) Ferroli P, Acerbi F, Tringall G, Albanese E, Broggi M, Franzini A, Broggi $G$ : Venous sacrifice in neurosurgery : new insights from venous indocyanine green videoangiography. $J$ Neurosurg 115: 18-23, 2011.

8) Gailloud P, San Millán Ruíz D, Muster M, Murphy J, Fasel JHD, Rüfenacht DA : Angiographic anatomy of the laterocavernous sinus. AJNR Am J Neuroradiol 21 : 1923-1929, 2000.

9) Hirsch JF, Zouaoui A, Renier D, Pierre-Kahn A : A new surgical approach to the third ventricle with interruption of the striathalamic vein. Acta Neurochirur (Wien) 47:135-147, 1979.

10) Houkin $K$, Takikawa $S$, Sawamura $Y$, Saitoh $H$, Abe $H$ : Intracranial vein reconstruction. in Hakuba A(ed) : Surgery of the 
Intracranial Venous System : Embryology, Anatomy, Pathophy Siology, Neuroradiology, Diagnos Treatment. New York, Springer, 1996, pp.375-379.

11) Kempe LG : Operative Neurosurgery Vol. 2 : Posterior Fossa, Spinal Cord and Peripheral Nerve Disease. New York, Springer-Verlag, 1970, pp.34-45.

12) Kodera T, Bozinov O, Sürücü O, Ulrich NH, Burkhardt JK, Bertalanffy $\mathrm{H}$ : Neurosurgical venous considerations for tumors of the pineal region resected using the infratentorial supracerebellar approach. J Clin Neurosci 18:1481-1485, 2011.

13) Koerbel A, Wolf SA, Kiss A: Peduncular hallucinosis after sacrifice of veins of the petrosal venous complex for trigeminal neuralgia. Acta Neurochir(Wien) $149: 831-833,2007$.

14) Komiyama $M$ : Essential anatomical knowledge for neurointervention : functional neurovascular anatomy. Jpn J Neurosurg (Tokyo) 13:116-125, 2004.

15) Komiyama $\mathrm{M}:$ Functional anatomy of the cerebral veins. Jpn J Neurosurg (Tokyo) 18:821-829, 2009.

16) Komiyama $M:$ History of angiography of the cerebral and spinal vessels and the importance of stereoscopic viewing. JNET 9:5-15, 2015.

17) Komiyama M : Cerebrovascular diseases from the perspective of embryology, anatomy and genetics. No Shinkei Geka 45: 103-115, 2017.

18) Krause F : Operative Freilegung der Vierhügel, nebst Beobachtungen über Hirndruck und Dekompression (mit Lichtbildern). Zentralbl Chir $53: 2812-2819,1926$.

19) Malis LI : Venous involvement in tumor resection. in Hakuba A (ed) : Surgery of the Intracranial Venous System: Embryology, Anatomy, Pathophy Siology, Neuroradiology, Diagnos Treatment. New York, Springer, 1996, p.281-288.

20) Masuoka J, Matsushima T, Hikata T, Inoue E : Cerebellar swelling after sacrifice of the superior petrosal vein during microvascular decompression for trigeminal neuralgia. $J$ Clin Neurosci $\quad$ 16:1342-1344, 2009.

21) McKissock $W$ : The surgical treatment of colloid cyst of the third ventricle; a report based upon twenty-one personal cases. Brain 74: 1-9, 1951.

22) McLaughlin MR, Jannetta PJ, Clyde BL, Subach BR, Comey $\mathrm{CH}$, Resnick DK : Microvascular decompression of cranial nerves : lessons learned after 4400 operations. J Neurosurg 90: 1-8, 1999.

23) McLaughlin MR, Jannetta PJ, Subach BR, Clyde BL : Coagulation of the petrosal vein for MVD. (letter). J Neurosurg 90: 1148, 1999.

24) Morita A, Sekhar LN : Reconstruction of the vein of Labbè by using a short saphenous vein bypass graft. Technical
note.J Neurosurg 89:671-675, 1998.

25) Nakase H, Shin Y, Nakagawa I, Kimura R, Sakaki T : Clinical features of postoperative cerebral venous infarction. Acta Neurochir (Wien) 147:621-626, 2005.

26) Otsuka H, Nakase H, Nagata K, Ueda K, Kempski O, Sakaki $\mathrm{T}$ : Effects of age on cerebral venous circulation disturbances in the rat. J Neurosurg $93: 298-304,2000$.

27) Page LK: The intratentorial-supracerebellar exposure of tumors in the pineal area. Neurosurgery $1: 36-40,1977$.

28) Pendl G: Infratentorial approach to mesencephalic tumors. in Koos WT, Bock FW, Spetzler RF (eds) : Clinical Microneurosurgery. Studttgart, Georg Thieme, 1976, pp.143-150.

29) Rhoton AL Jr: The posterior fossa veins. Neurosurgery 47 (3 Suppl) : S69-S92, 2000.

30) Sakaki T, Morimoto T, Takemura K, Miyamoto S, Kyoi K, Utsumi S : Reconstruction of cerebral cortical veins using silicone tubing. Technical note. J Neurosurg $66: 471-473$, 1987.

31) Savardekar AR, Goto T, Nagata T, Ishibashi K, Terakawa Y, Morisako H, Ohata K : Staged 'intentional' bridging vein ligation : a safe strategy in gaining wide access to skull base tumors. Acta Neurochir (Wien) 156:671-679, 2014.

32) Schlesinger B : The tolerance of the blocked Galenic system against artificially increased intravenous pressure. Brain $63: 178-183,1940$.

33) Seeger W : Microsurgery of Cerebral Veins. Wien, SpringerVerlag, 1984.

34) Sindou M, Auque J: The intracranial venous system as a "neurosurgeon's" perspective. Adv Tech Stand Neurosurg 26: 131-216, 2000 .

35) Sindou M, Auque J, Jouanneau E : Neurosurgery and the intracranial venous system. Acta Neurochir Suppl 94: 167-175, 2005.

36) Stein BM : The infratentorial supracerebellar approach to pineal lesions. J Neurosurg $35:$ 197-202, 1971.

37) Strauss C, Neu M, Bischoff B, Romstöck J : Clinical and neurophysiological observations after superior petrosal vein obstruction during surgery of the cerebellopontine angle: case report. Neurosurgery 48: 1157-1161, 2001.

38) Sugita K, Kobayashi S, Yokoo A : Preservation of large bridging veins during brain retraction. Technical note. J Neurosurg 57: 856-858, 1982.

39) Tsukamoto H, Matsushima T, Fujiwara S, Fukui M : Peduncular hallucinosis following microvascular decompression for trigeminal neuralgia : case report. Surg Neurol $\quad 40$ : $31^{-}$ 34, 1993.

40) Yamamoto I, Kageyama N : Microsurgical anatomy of the pineal region. J Neurosurg $53:$ 205-221, 1980. 